\title{
Down regulating PHGDH affects the lactate production of sertoli cells in varicocele
}

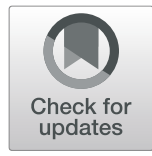

Wen-bin Guo ${ }^{\dagger}$, Zhen-hui Huang ${ }^{\dagger}$, Cheng Yang ${ }^{\dagger}$, Xian-yuan Lv, Hui Xia, Hu Tian, Jian-kun Yang, Qi-zhao Zhou, Ming-kun Chen, Kang-yi Xue and Cun-dong Liu*

\begin{abstract}
Background: Although varicocele is considered to be one of the leading causes of male infertility, the precise mechanism underlying how varicocele leads to male infertility is not completely understood. We found the lactate concentration on the varicocele side of the patients was decreased compare with peripheral venous blood. In the testicles, the lactate produced by the sertoli cells through the glycolysis pathway provides most of the energy needed for spermatogenesis, the reduction of lactate will affect spermatogenesis. The objective of this study was to investigate the mechanism of this abnormal energy metabolism phenomenon in varicocele.
\end{abstract}

Methods: In this study, we collected the testicular tissue from patients with varicocele, the glycolysis related proteins PHGDH was identified by iTRAQ proteomics technology. Experimental rat varicocele model was constructed according to our new clip technique, the mRNA and protein expression levels of PHGDH were examined with GRT-PCR and Western blotting. We constructed a sertoli cell of PHGDH down-regulation model, and then detected the glucose consumption, LDH activities and lactate production in the sertoli cells. Western blot was conducted to investigate the effects of PHGDH on the expression of phosphoserine phosphatase (PSPH) and Pyruvate kinase M2 (PKM2). Flow cytometry was used to detect the cell apoptosis and cell cycle in sertoli cells.

Results: The results showed that testicular protein PHGDH was down-regulated in patients with varicocele and in experimental rat varicocele model. Down-regulation of PHGDH in sertoli cells significantly decreased the glucose consumption, LDH activities and lactate production in the sertoli cells, indicating that the low expression of PHGDH ultimately led to a decrease in lactate production by affecting the glycolysis. The Western blot results showed that the down-regulation of PHGDH significantly reduced the expression of pathway protein PSPH and PKM2, leading to the reduction of lactate production. Moreover, PHGDH knockdown can promote apoptosis and inhibit cell cycle to affect cell growth.

Conclusions: Overall, we conformed that varicocele lead to the decreasing of testis lactate production. Downregulation of PHGDH in sertoli cells may mediate the process of abnormal glucose metabolism. Our study provide new insight into the mechanisms underlying metabolism-associated male infertility and suggests a novel therapeutic target for male infertility.

Keywords: Varicocele, Phosphoglycerate dehydrogenase (PHGDH), Glycolysis, Lactate metabolism, Sertoli cells (SC)

\footnotetext{
* Correspondence: cundongliu@163.com

'Wen-bin Guo, Zhen-hui Huang and Cheng Yang contributed equally to this work.

Department of Urology, the Third Affiliated Hospital of Southern Medical

University, Guangzhou, China
}

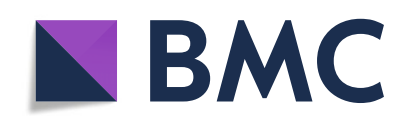

(c) The Author(s). 2020 Open Access This article is licensed under a Creative Commons Attribution 4.0 International License, which permits use, sharing, adaptation, distribution and reproduction in any medium or format, as long as you give appropriate credit to the original author(s) and the source, provide a link to the Creative Commons licence, and indicate if changes were made. The images or other third party material in this article are included in the article's Creative Commons licence, unless indicated otherwise in a credit line to the material. If material is not included in the article's Creative Commons licence and your intended use is not permitted by statutory regulation or exceeds the permitted use, you will need to obtain permission directly from the copyright holder. To view a copy of this licence, visit http://creativecommons.org/licenses/by/4.0/ The Creative Commons Public Domain Dedication waiver (http://creativecommons.org/publicdomain/zero/1.0/) applies to the data made available in this article, unless otherwise stated in a credit line to the data. 


\section{Background}

Varicocele, the abnormal dilation and tortuosity of the pampiniform venous plexus within the spermatic cord, is present in about $15 \%$ of the male adult population and almost $40 \%$ of infertile males [1]. Many factors account for male infertility in varicocele, such as hypoxia, metabolic abnormalities, hormonal dysfunction, elevated testicular temperature and spermatic veins hypertension [2]. The precise mechanism by which varicocele might cause infertility is still unknown. No single factor is believed to be responsible for the negative testicular effects [3]; instead the pathogenesis is believed to be complex and multifactorial, with several proposed mechanisms acting together [4]. In this complex pathophysiological network, metabolic abnormalities seems to have a important role, although some studies have suggested a link between abnormal glycolysis and varicocele, the specific mechanisms are still poorly understood [5].

Sertoli cells, located in the basal compartment of seminiferous tubules, often referred to as nurse cells, are responsible for providing energy and nutritional support to developing germ cells [6]. It is imperative that germ cells receive an adequate level of energy substrates, otherwise they will degenerate and enter the apoptotic pathway [7]. In fact, the majority of energy required for spermatogenesis are provided by sertoli cells through glycolysis to produce lactate [8]. Therefore, the glycolysis of sertoli cells defines the population size of germ cells, which is essential for the maintenance of spermatogenesis and consequently, male fertility [9].

PHGDH, the first enzyme branching from glycolysis in a three-step serine biosynthetic pathway uses NAD+ as a cofactor to oxidize 3-phosphoglycerate into phosphohydroxypyruvate. The product is then subsequently converted into phosphoserine via transamination by PSAT1 and, ultimately, to serine via phosphate ester hydrolysis and the enzyme PSPH [10], serine is also an activator of PKM2 in the glycolysis [11]. The overexpression of PHGDH is often associated with progression of cancers, and the inhibitors of PHGDH reduce the glycolysis and suppress the growth of cancers [12]. However, the associated investigation in the reproductive system remains limited.

In this study, We found for the first time that the lactate on the varicocele side of the patients decreased and testicular protein $\mathrm{PHGDH}$ was down-regulated in varicocele. We revealed that varicocele lead to the low expression of PHGDH in sertoli cells and the low expression of PHGDH ultimately led to a decrease in lactate production by affecting the glycolysis pathway. Moreover, PHGDH knockdown can promote apoptosis and inhibit cell cycle to affect cell growth. That may eventually lead to impairment of spermatogenesis and male infertility. This may help to better understand the major proteins contributing to male infertility in varicocele, further exploring the mechanisms underlying male infertility, and developing novel treatments for varicocele.

\section{Methods \\ Blood gas analysis in patients with varicocele}

During laparoscopic varicocelectomy, after exposing the testicular vein, Venous blood is extracted with a syringe with a slender needle before high ligation, and the scrotum is squeezed as the blood is extracted. Finally, blood gas analysis was performed on the varicocele side venous blood and peripheral venous blood of patients at the same time to detect lactate concentration. This study was approved by the ethics committee of the Third Affiliated Hospital of Southern Medical University under code number 68222115 .

\section{The establishment and assessment of the experimental rat varicocele model}

Twenty adult male specific-pathogen free SpragueDawley rats (body weight, 250-300 g) were randomly assigned to a sham group $(n=10)$ and a varicocele group $(n=10)$. Rats were housed in a climate-controlled environment with free access to food and water under a 12-h day/night cycle. Experimental rat varicocele model was constructed according to our new clip technique [13]. The establishment and assessment of the rat model is described in the previous articles published by our team. This study was approved by the ethics committee of the Third Affiliated Hospital of Southern Medical University and the animal work was approved by the Animal Care and Ethics Committee of Southern Medical University.

\section{RNA isolation and qRT PCR analysis}

Total RNA was extracted from the left testis of each animal using TRIzol reagent (Invitrogen, Carlsbad, USA) in accordance with the manufacturer's instructions. In brief, tissues were homogenized and incubated in TRIzol for $10 \mathrm{~min}$, and then RNA was separated using chloroform $(0.2 \mathrm{~mL} / 1 \mathrm{~mL}$ TRIzol). After centrifugation at 12, $000 \mathrm{~g}$ for $15 \mathrm{~min}$ at $4{ }^{\circ} \mathrm{C}$, the aqueous phase of the sample was transferred to a fresh tube for RNA precipitation using isopropyl alcohol $(0.5 \mathrm{~mL} / 1 \mathrm{~mL}$ TRIzol). After repeating the incubation and centrifugation steps, the remaining pellet was washed with $75 \%$ ethanol and centrifuged at $7500 \mathrm{~g}$ for $5 \mathrm{~min}$ at $4{ }^{\circ} \mathrm{C}$. Finally, the air-dried pellet was re-dissolved in RNase-free water. The quantity and quality of the extracted RNA were measured with ESpect (Malcon, Japan). In total, 200 ng of total RNA was reverse-transcribed with a PrimerScriptTM RT Kit (TaKaRa) for mRNA while quantitative real-time PCR for mRNA was performed in a 96-well plate using a SYBR Premix Ex Taq Real Time PCR Kit (TaKaRa). 
Amplification reactions were carried out in a final volume of $20 \mu \mathrm{L}$ and were performed on an Mx3005P Stratagene under the following thermal cycling conditions: (denaturation at $95^{\circ} \mathrm{C}$ for $30 \mathrm{~s}[1 \times]$, followed by 40 cycles of denaturation $\left[95^{\circ} \mathrm{C}, 30 \mathrm{~s}\right]$, annealing $\left[60^{\circ} \mathrm{C}\right.$, $10 \mathrm{~s}$ ] and extension $\left.\left[72^{\circ} \mathrm{C}, 15 \mathrm{~s}\right]\right)$. Rat Gapdh was used as an endogenous reference. The primers used for detecting Phgdh and Gapdh expression are shown in Table 1. The comparative cycle threshold method was performed for relative quantification. The sequences of the primers were as follows: PHGDH forward, 5' - GATGAAAGAT GGCAAATGGGA -3'; PHGDH reverse, 5' - GCGGGG TATGGACAGTGATG $-3^{\prime}$. GAPDH forward, $5^{\prime}$ TGGAGTCTAGGCGTCTT -3'; GAPDH reverse5'TGTCATATTTCTCGTGGTTCA - 3'.

\section{Cell culture}

Sertoli cells (a mouse testis Sertoli cell line) were purchased from American Type Culture Collection (ATCC, Manassas, VA, USA) and cultured in DMEM with $10 \%$ FBS at $37{ }^{\circ} \mathrm{C}$ in an incubator with an atmosphere of $5 \%$ $\mathrm{CO} 2$. For the experiments, sertoli cells were adherently cultured in 100-mm tissue culture dishes and reached $60 \sim 70 \%$ density before use.

\section{Small interfering RNA (siRNA) and transient transfection} PHGDH siRNA was used to silence the PHGDH gene. A scrambled sequence siRNA (siNCtrl) was used as a negative control. The siRNA transfection was optimized using Lipofectamine2000-RNAimax (Invitrogen, Carlsbad, CA, USA), according to the manufacturer $\mathrm{s}$ instructions. Briefly, siRNA and lipofectamine were diluted separately in Opti-MEM (Gibco) and incubated at room temperature for $5 \mathrm{~min}$. Then, the two solutions were gently mixed and incubated for $15 \mathrm{~min}$. Finally, the mixture was added to plated cells, and after $48 \mathrm{~h}$, the cells were analyzed using the following assays.

\section{Flow cytometry for cell apoptosis and cell cycle}

PHGDH siRNA and a negative control siRNA were transfected as mentioned above.For cell apoptosis analysis, cells were prepared with the PE Annexin V Apoptosis Detection Kit I (BD Biosciences, New Jersey, USA) according to the manufacturer's recommendations. For cell cycle analysis, cells were fixed and permeabilized by $75 \%$ ethanol, and were stained by PI/RNase Staining Buffer (BD Biosciences, New Jersey, USA) after incubation at $-20^{\circ} \mathrm{C}$ overnight. The cell apoptosis ratio and cell cycle profile were

\begin{tabular}{lll} 
Table $\mathbf{1}$ The primers used for the real-time PCR \\
\hline Gene & Forward Primer (5-3) & Reverse Primer (5-3) \\
GAPDH & TGGAGTCTACTGGCGTCTT & TGTCATATTTCTCGTGGTTCA \\
PHGDH & GATGAAAGATGGCAAATGGGA & GCGGGGTATGGACAGTGATG \\
\hline
\end{tabular}

detected by FAC Station (FV500,Beckman Coulter, Brea, USA), and the raw data were analyzed using FlowJo 10.0.7 software (FlowJo, Oregon, USA).

\section{Western blot analysis}

Total cells washed twice with cold PBS and lysed with RIPA (lysis buffer radioimmunoprecipitation assay) (Beyotime Institute of Biotechnology, China) and PMSF (protease inhibitors phenylmethanesulfonyl fluoride) (Beyotime Institute of Biotechnology, China). The protein concentration was determined by utilizing bicinchoninic acid (BCA) protein assay kit (P0010S, Beyotime Biotech, China). Equal amounts of protein $(\sim 10 \mu \mathrm{g})$ were separated by $10 \%$ sodium dodecyl sulfate polyacrylamide gel electrophoresis (SDS-PAGE) and then transferred to $0.45 \mu \mathrm{m}$ polyvinylidine difluoride filter (PVDF) membranes. The membranes were blocked with $5 \%$ BSA for $1 \mathrm{~h}$ and then the membranes were incubated at $4{ }^{\circ} \mathrm{C}$ overnight with primary antibodies. Next, the membranes were washed three times with TBST for at least $15 \mathrm{~min}$, and probed with HRP linked secondary antibodies for 1 $\mathrm{h}$ at room temperature. Protein bands were visualized employing the enhanced chemiluminescence detection kit (Thermo scientific, USA). The gray value of each protein band was analyzed by Image Lab software.

The primary antibodies were: PHGDH,PKM-2,PSPH and $\beta$-actin. All these antibodies were purchased from Abcam.

\section{Glucose consumption and lactate production}

PHGDH siRNA and a negative control siRNA were transfected as mentioned above.After $48 \mathrm{~h}$, the media were collected for measurement of glucose and lactate concentrations as determined by glucose (GO) assay kit (Sigma) and lactate assay kit (Biovision). Glucose consumption and lactate production were normalized by cell numbers.

\section{Measurement of LDH activities}

PHGDH siRNA and a negative control siRNA were transfected as mentioned above. After $48 \mathrm{~h}$, the media were collected for measurement of LDH activities as determined by colorimetric assay kits in accordance with the manufacturer protocols.

\section{Protein-protein interaction (PPI) network construction}

Using the Search Tool for the Retrieval of Interacting Genes (STRING) database (http://www.string-db.org/), a PPI network related to PHGDH, PSPH, PSAT1 and PKM2 was established. The interactions procured included known interactions and predicted interactions. 


\section{Statistical analysis}

Data were statistically analyzed using GraphPad Prism 8.0 software, and were presented as the mean \pm standard deviation (SD). An unpaired t-test was used to analyze the differences between the two groups. One-way ANOVA was used to analyze the intergroup differences among multiple groups. $P<0.05$ was considered statistically significant.

\section{Results}

The lactate on the varicocele side of the patients decreased

The blood gas analysis results showed that compared with the peripheral venous blood, the lactate concentration in the varicocele side of the patients decreased significantly (Fig. 1).

\section{Testicular protein PHGDH was down-regulated in varicocele}

PHGDH was of interest because of its involvement in lactic acid metabolism, and we chose to test our hypothesis in a rat model. Eight weeks after initial surgery, the mean diameter of the LSV in the varicocele group was significantly larger than that of the sham group $(1.58 \pm$ $0.05 \mathrm{~mm}$ versus $0.24 \pm 0.01 \mathrm{~mm}$, respectively, $P<0.0001$, Supplementary Fig. 1). Histological assessment of the left testes showed that the proportion (\%) of degenerating seminiferous tubules was significantly higher in the varicocele group than in the sham group $(57.83 \pm 3.63 \%$ versus $13.73 \pm 0.65 \%$, respectively, $P<0.0001$, Fig. $2 \mathrm{a}, \mathrm{b}$ ), which indicated clear impairment of spermatogenesis. The mRNA and protein expression levels of PHGDH were examined with qRT-PCR and Western blotting, respectively, in the left testes from the two groups of rats.

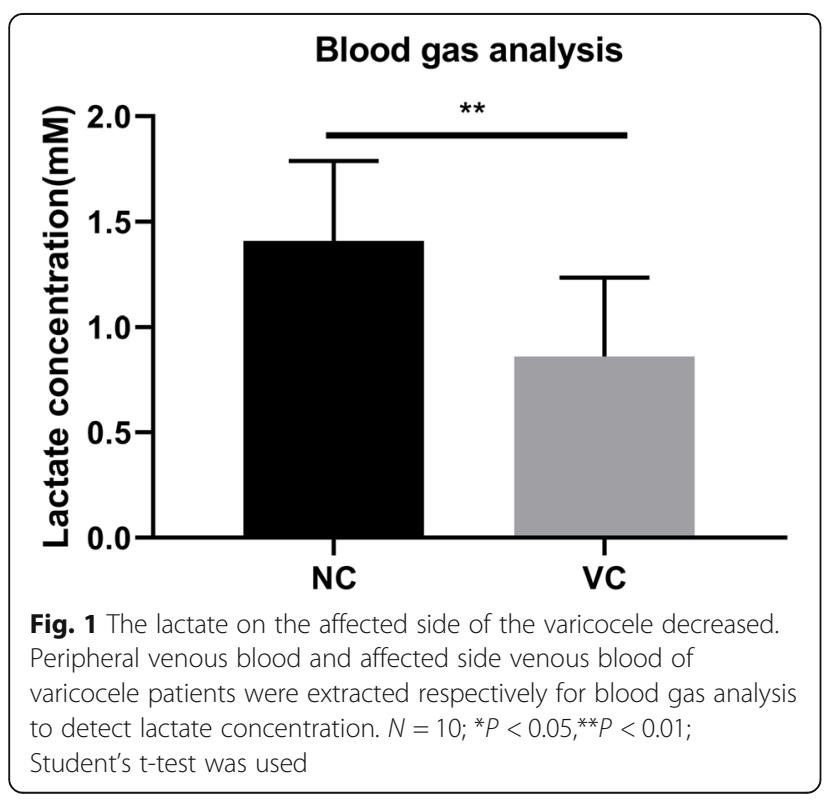

Notably, PHGDH mRNA and protein expression was obviously reduced in the left testes from the varicocele group relative to the sham group (Fig. 2c, d).

\section{PHGDH knockdown inhibited sertoli cells glycolysis and lactate production}

In order to investigate the role of PHGDH in sertoli cells, the expression of PHGDH was knocked down by PHGDH siRNA. sertoli cells were transfected with a scrambled sequence siRNA (siNCtrl) and three PHGDH siRNA. the protein level of PHGDH were inhibited significantly by PHGDH siR1 (Fig. 3a, b). Because the production of lactate to fulfill the energy needs of spermatogenesis is a crucial function of sertoli cells, and PHGDH may cause changes in cell lactate production by affecting glycolysis, we wondered whether PHGDH Knockdown regulate sertoli cells glycolysis. After transiently transfection sertoli cells with NC siR or PHGDH siR1 for $48 \mathrm{~h}$, PHGDH knockdown significantly decreased the glucose consumption (Fig. 3c) and the LDH activities (Fig. 3d) of sertoli cells. We further examined the lactate production of sertoli cells, which showed that PHGDH knockdown inhibited the lactate production of sertoli cells (Fig. 3e). These results indicate that PHGDH knockdown inhibited the glycolysis of sertoli cells and resulted in the reduction of lactate production.

\section{PHGDH regulates the expression of PSPH and PKM2 expression in sertoli cells}

To investigate the mechanism how PHGDH regulates sertoli cell glycolysis and lactate production, we constructed a PPI network using the STRING database, It was verified that PSPH, PSAT1 and PKM2 were indeed related to PHGDH (Fig. 4a). We performed western blot to access the effects of PHGDH on the expression of PSPH and PKM2. Our results showed that knockdown of PHGDH remarkably decreased the expression of PSPH and PKM2 (Fig. 4b).

\section{PHGDH knockdown inhibited sertoli cells growth}

After transiently transfection sertoli cells with $\mathrm{NC}$ siR or PHGDH siR1 for $48 \mathrm{~h}$, cell cycle and cell apoptosis assays indicated that PHGDH knockdown decreased the proportion of cells in S-phase (Fig. 5a) and increased the percentage of apoptotic cells (Fig. 5b) in sertoli cells compared with control groups. These results indicate that PHGDH might play a key role in sertoli cells growth.

\section{Discussion}

Although varicocele is considered to be one of the leading causes of male infertility [14], the precise mechanism underlying how varicocele leads to male infertility is not completely understood. As early as 1981, a study found that the production of lactate in the varicocele side of 


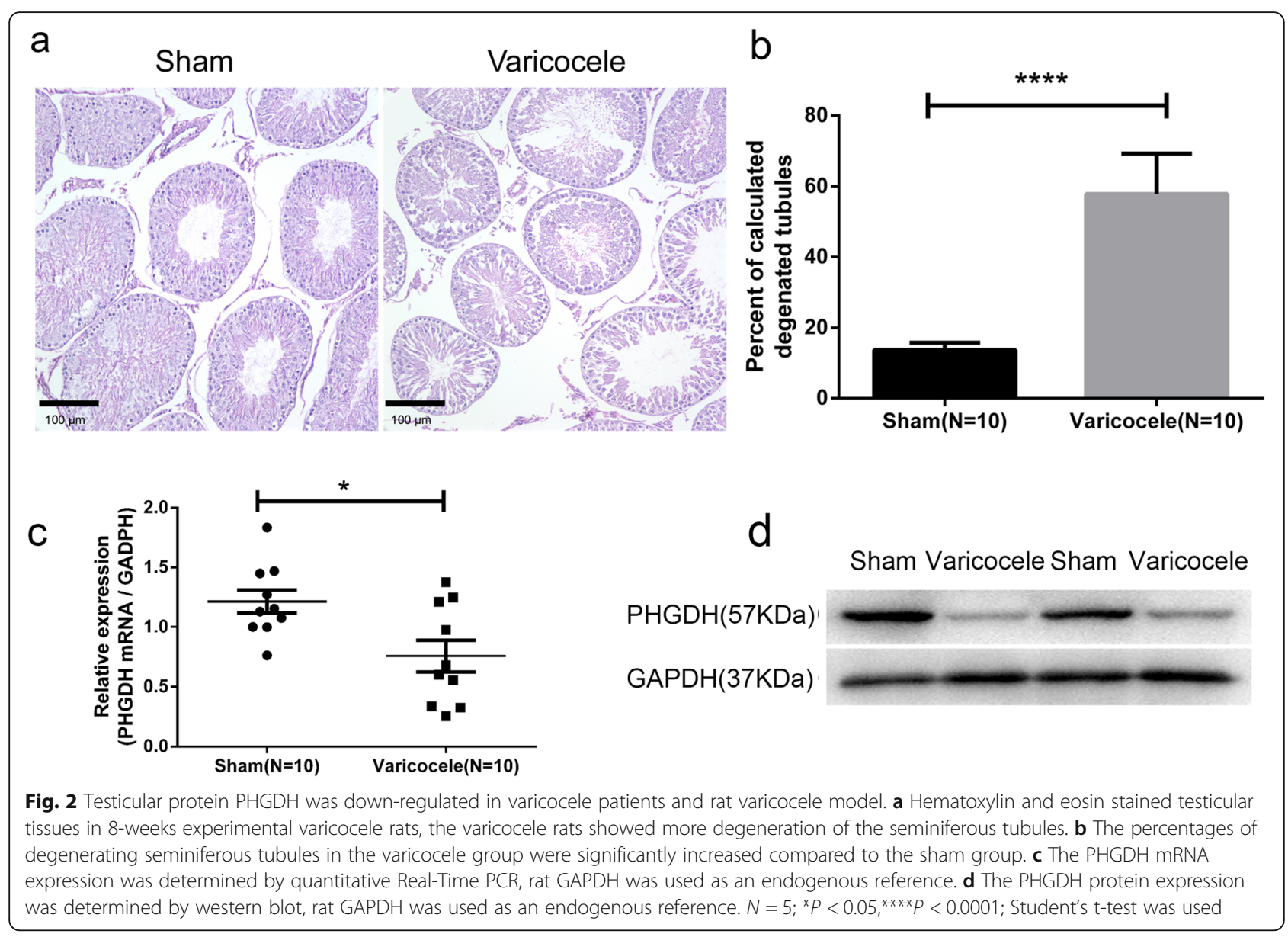

the patients was decreased, which was speculated to be caused by the absence of glycolysis [15]. Another study detected a decrease in the level of lactate in spermatic plasma in infertile patients with varicocele [16]. Using the varicocele model of rabbit, it was found that the single spermatic vein could cause the decrease of lactate and pyruvate in both spermatic veins [17]. In the above studies, it was found that varicocele could cause the decrease of lactate in the varicocele side and the seminal plasma. In our study, we collected the blood in the varicocele side and the peripheral venous blood of the patients for blood gas analysis, found that the lactate on the varicocele side of the patients showed a decrease. In the testicles, lactate is mainly generated by sertoli cells through the glycolysis pathway, which supplies energy to spermatogonial cells and maintains their proliferation and differentiation [18]. The reduction of lactate will affect spermatogenesis [8]. Therefore, this clinical phenomenon has aroused our interest.

To investigate the mechanism behind the reduction of lactate, we collected a small amount of testicular tissue from patients with varicocele and found that the glycolysis related proteins PHGDH was down-regulated in varicocele patients (Supplementary File1). Due to the small sample size, we chose to verify the results in experimental rat varicocele model. Clearly, the current surgicallyinduced varicocele rat model differs from the situation seen in clinical patients with varicocele in several respects, such as venous anatomy, and the duration of varicocele. However, the rat model is widely accepted and used for investigating the pathophysiology of varicocele [19]. Finally, we found that testicular protein PHGD $\mathrm{H}$ was down-regulated in varicocele patients and rat varicocele model, the decrease of PHGDH expression may be the reason for the decrease of lactate.

PHGDH, encoding 3-phosphoglycerate dehydrogenase, is located on chromosome $1 \mathrm{p} 12$ and is widely distributed in organisms and in tissues [20]. PHGDH is the frst enzyme branching from glycolysis in a three-step serine biosynthetic pathway uses NAD+ as a cofactor to oxidize 3phosphoglycerate into phosphohydroxypyruvate. The product is then subsequently converted into phosphoserine via transamination by phosphoserine aminotransferase (PSAT1) and, ultimately, to serine via phosphate ester hydrolysis and the enzyme PSPH, serine is also an activator of PKM2 in the glycolysis. Over recent years, an increasing number of studies have focused upon PHGDH in cancer research, which is found to exhibit elevation and 


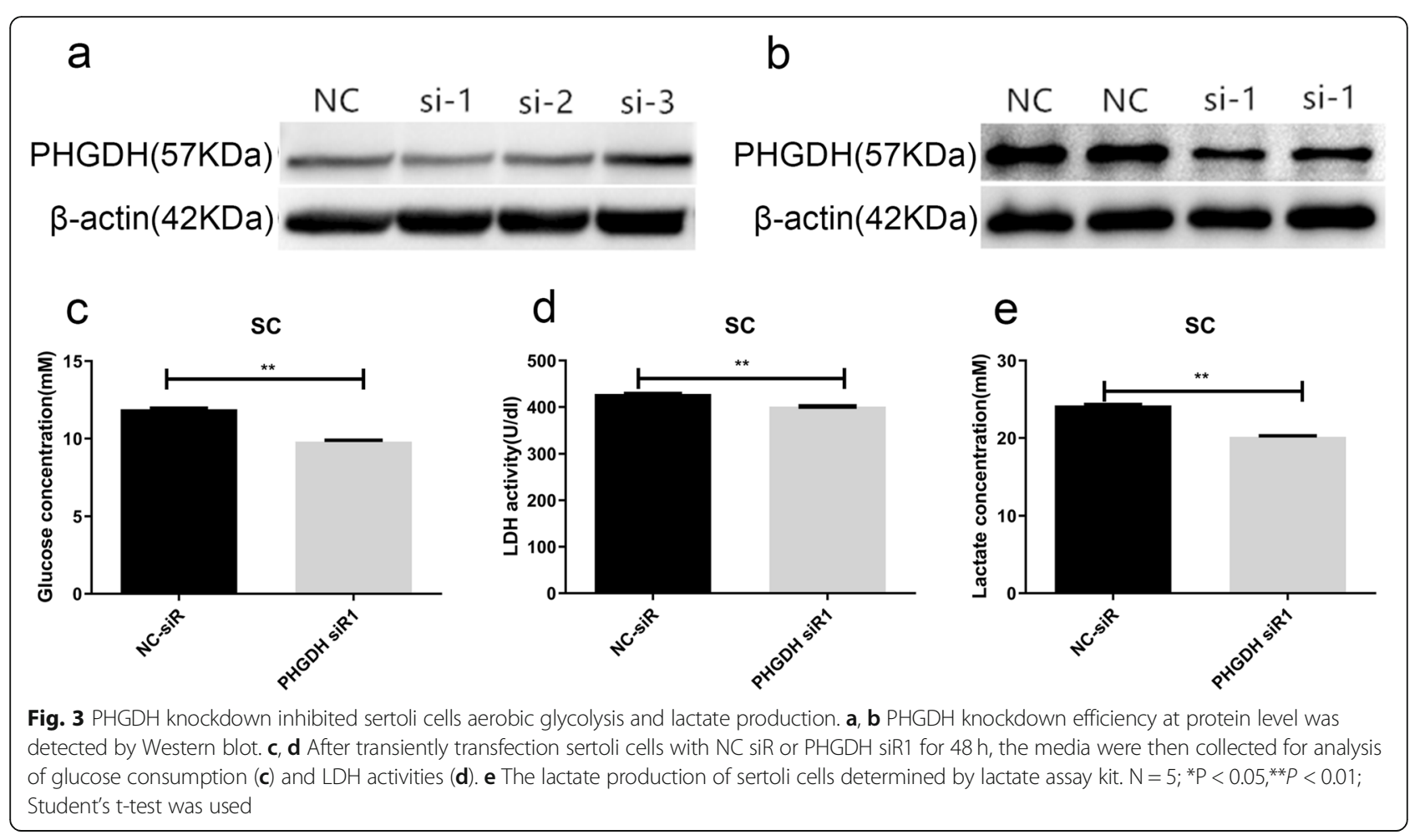

controlled flux throughout the serine biosynthetic pathway in cancer cells [21]. Interestingly, The overexpression of PHGDH is often associated with progression of cancers, and the inhibitors of PHGDH reduce the glycolysis and suppress the growth of cancers [22]. PHGDH amplification may alter glucose metabolism in human melanoma, and may result in changes in redox status, energy metabolism, and potentially, other signaling functions [10]. These observations suggested that PHGDH was implicated in cell growth, proliferation and glycolysis. PHGDH mRNA was found to be expressed at high levels in testicular tissue [20], and PHGDH was considered as testicular spermatogenesis-related panels [23]. PHGDH was also shown to be major antigen for ovarian autoimmunity associated with female infertility [24]. However, the associated investigation in the reproductive system remains limited.

In order to investigate the role of PHGDH in sertoli cells, we constructed a sertoli cell of PHGDH downregulation model. Down-regulation of PHGDH in sertoli cells significantly decreased the glucose consumption, LDH activities and lactate production in the sertoli cells, indicating that the low expression of PHGDH ultimately led to a decrease in lactate production by affecting the

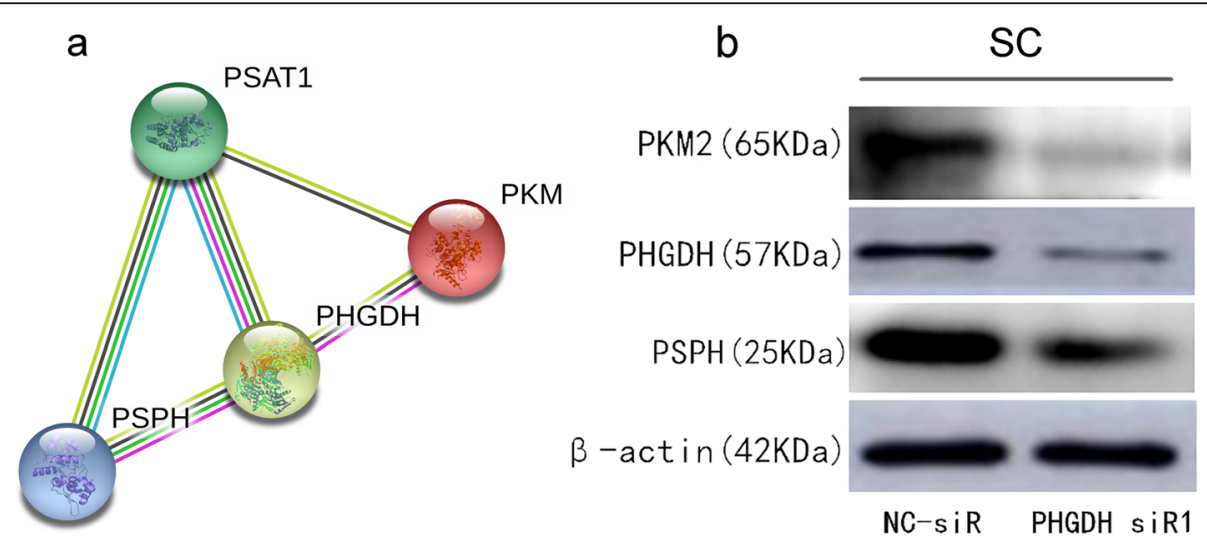

Fig. 4 PHGDH regulates the expression of PSPH and PKM2 expression in sertoli cells. a PPI network showed that PSPH, PSAT1 and PKM2 were indeed correlated with PHGDH. b The protein expression levels of PSPH and PKM2 in PHGDH-siRNA transfected cells and empty vectortransfected cells were examined by western blot analysis, Western blot experiment was repeated three separate times 


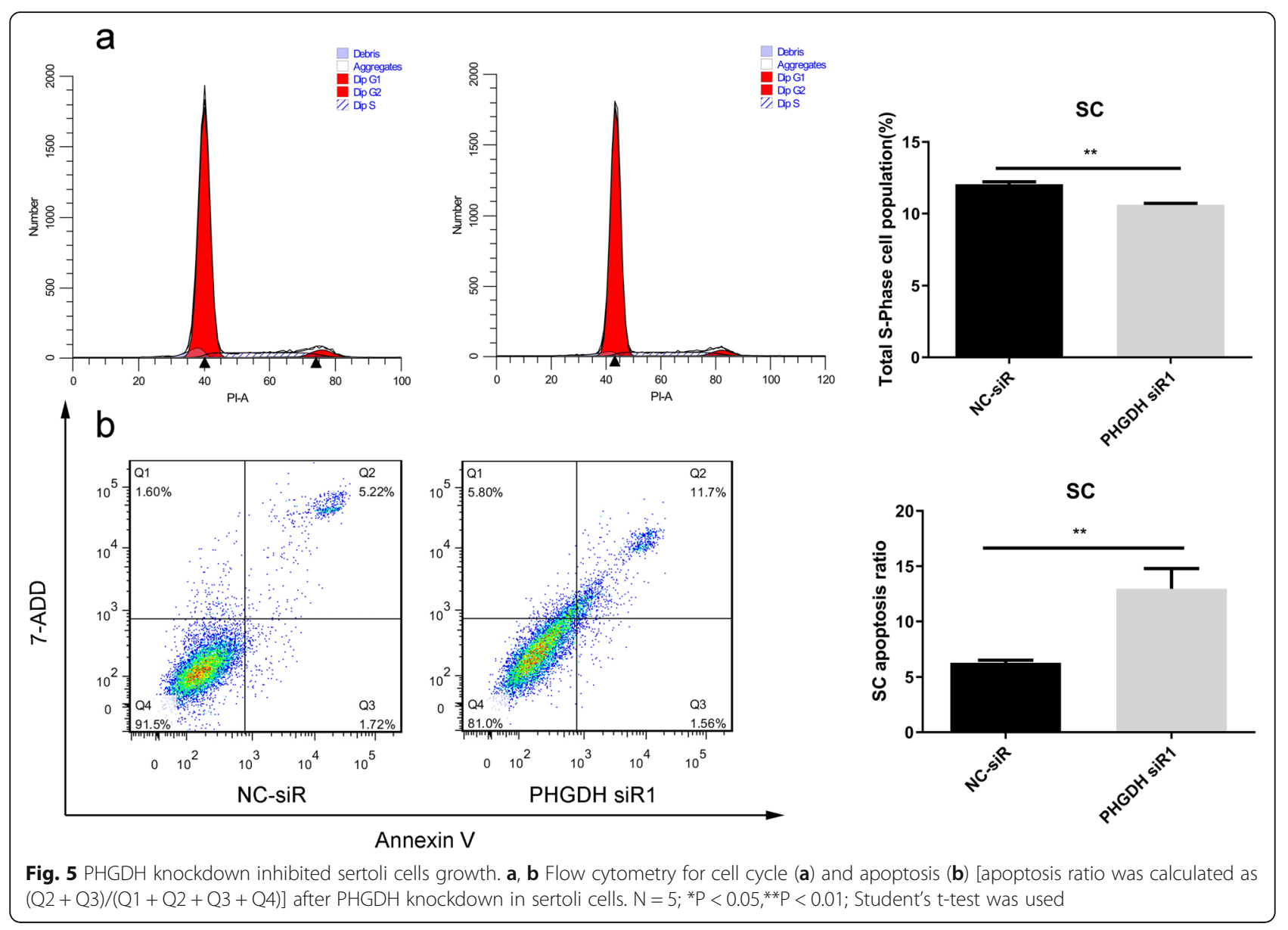

glycolysis. Western blot was conducted to investigate the effects of PHGDH on the expression of PSPH and PKM2, and the results showed that the down-regulation of PHGDH significantly reduced the expression of pathway protein PSPH and PKM2, leading to the reduction of lactate production.

Autophagy is considered to be a process conserved during evolution that plays an important role in physiological and pathological conditions. Its main role is the degradation of harmful cytoplasmic components such as damaged organelles and poorly folded proteins that are no longer needed. In different diseases, the relationship between autophagy and apoptosis is not the same [25]. Study found that autophagy can aggravate the apoptosis of malignant glioma cells [26]. Another study found that autophagy could improve the ability of cells to fight infection, which reduces the apoptosis of infected cells [27]. And previous studies have shown that in varicocele, the decrease of glycolysis products is an important cause of germ cell autophagy, and long-term autophagy increases germ cell apoptosis [5]. Our results showed that PHGDH knockdown can promote apoptosis and inhibit cell cycle to affect sertoli cell growth. In fact, many previous studies have shown that varicocele activates apoptosis in seminiferous epithelial cells leading to sertoli-mediated phagocytosis of apoptotic germ cells [28]. These studies suggests that autophagy induced by long-term hypoxia in varicocele is an important cause of apoptosis [29]. Our study suggests that the decrease in glycolysis products due to low expression of PHGDH may also be an important cause of apoptosis in varicocele. It may also lead to abnormal testicular energy metabolism in patients with varicocele, and ultimately affect spermatogenesis and lead to infertility. This may help to better understand the major proteins contributing to male infertility in varicocele, further exploring the mechanisms underlying male infertility, and developing novel treatments for varicocele.

\section{Conclusions}

In summary, our current findings indicate that varicocele lead to the low expression of PHGDH in sertoli cells and the low expression of PHGDH ultimately led to a decrease in lactate production by affecting the glycolysis pathway. Moreover, PHGDH knockdown can promote apoptosis and inhibit cell cycle to affect cell growth. That may be one of the important causes of impairment of spermatogenesis and male infertility. 
Nevertheless, there are some limitations to this study. First, we discovered that testicular protein PHGDH was down-regulated in varicocele male adults with asthenospermia (Supplementary File1), due to the small sample size, we chose to verify the results in experimental rat varicocele model. Second, we did not further investigate the effect of lactate reduction on the proliferation and differentiation of spermatogonia, and is therefore under progress in our lab.

At present, the literature relating to PHGDH in spermatogenesis is quite limited and many questions, particularly related to mechanisms, still remain to be elucidated regarding the mechanisms underlying varicocele-related infertility. Our study provides a new viewpoint to reveal the mechanisms underlying metabolism-associated male infertility. Moreover, these data highlight that PHGDH is a potential modulatory biomarker in varicocele, which may help in the development of a new therapeutic target for male infertility.

\section{Supplementary information}

Supplementary information accompanies this paper at https://doi.org/10. 1186/s12958-020-00625-9.

\section{Additional file 1. \\ Additional file 2 \\ Additional file 3}

\section{Abbreviations}

PHGDH: Phosphoglycerate dehydrogenase; PSPG: Phosphoserine phosphatase; PSAT1: Phosphoserine aminotransferase 1; PKM2: Pyruvate kinase M2; ANOVA: Analysis of variance; PPI: Protein-protein interaction; GO: Gene Ontology; iTRAQ: Isobaric tags for relative and absolute quantitation

\section{Acknowledgements}

The authors thank all the subjects who participated in this study. In addition, they thank all the technologists for their cooperation and contribution to this study.

\section{Authors' contributions}

$\mathrm{ZHH}$ participated in the design of the study, performed experiments, and drafted the manuscript. HX and JKY participated in acquisition of data and collection of clinical samples. MKC and QZZ analyzed and interpreted the experimental data. KYX participated in the design of the study and performed the statistical analysis. WBG participated in the revising of the manuscript and gave conceptual advice. CDL conceived of the study, and participated in its design and coordination and helped to draft the manuscript. All authors read and approved the final manuscript.

\section{Funding}

This study was supported in part by National Natural Science Foundation of China (81772257), Youth cultivation program of Southern Medical University (PY2018N076) and Medical Scientific Research Foundation of Guangdong Province (A2019557).

\section{Availability of data and materials}

The dataset supporting the conclusions of this article is included within the article.

\section{Ethics approval and consent to participate}

This study was approved by the bioethics committees of Third Affiliated Hospital of Southern Medical University, Guangzhou, China. Written informed consent was obtained from each healthy donor, and the experimental protocol was established according to the associated national guidelines from the Ministry of Science and Technology of China.

\section{Consent for publication}

All co-authors have seen and approved the final version of the paper and. have agreed to its submission for publication. All patients signed informed. written consent forms.

\section{Competing interests}

The authors declare that they have no conflict of interest.

Received: 4 February 2020 Accepted: 30 June 2020

Published online: 14 July 2020

\section{References}

1. Choi WS, Kim SW. Current issues in varicocele management: a review. World J Mens Health. 2013:31:12-20.

2. Sheehan MM, Ramasamy R, Lamb DJ. Molecular mechanisms involved in varicocele-associated infertility. J Assist Reprod Genet. 2014;31:521-6.

3. Gat Y, Zukerman Z, Chakraborty J, Gornish M. Varicocele, hypoxia and male infertility. Fluid mechanics analysis of the impaired testicular venous drainage system. Hum Reprod. 2005;20:2614-9.

4. Hassanin $\mathrm{AM}$, Ahmed $\mathrm{HH}$, Kaddah AN. A global view of the pathophysiology of varicocele. Andrology. 2018;6:654-61.

5. Sadeghi N, Erfani-Majd N, Tavalaee M, Tabandeh MR, Drevet JR, NasrEsfahani MH. Signs of ROS-associated autophagy in testis and sperm in a rat model of Varicocele. Oxidative Med Cell Longev. 2020;2020:5140383.

6. Rato L, Alves MG, Socorro S, Duarte Al, Cavaco JE, Oliveira PF. Metabolic regulation is important for spermatogenesis. Nat Rev Urol. 2012;9:330-8.

7. Boussouar $F$, Benahmed M. Lactate and energy metabolism in male germ cells. Trends Endocrinol Metab. 2004;15:345-50.

8. Oliveira PF, Martins AD, Moreira AC, Cheng CY, Alves MG. The Warburg effect revisited--lesson from the Sertoli cell. Med Res Rev. 2015;35:126-51.

9. Jutte NH, Grootegoed JA, Rommerts FF, van der Molen HJ. Exogenous lactate is essential for metabolic activities in isolated rat spermatocytes and spermatids. J Reprod Fertil. 1981;62:399-405.

10. Mullarky E, Mattaini KR, Vander Heiden MG, Cantley LC, Locasale JW. PHGDH amplification and altered glucose metabolism in human melanoma. Pigment Cell Melanoma Res. 2011;24:1112-5.

11. Chaneton B, Hillmann P, Zheng L, Martin AC, Maddocks OD, Chokkathukalam A, Coyle JE, Jankevics A, Holding FP, Vousden KHJN. Serine is a natural ligand and allosteric activator of pyruvate kinase M2. Nature. 2012;491:458.

12. Zogg CK. Phosphoglycerate dehydrogenase: potential therapeutic target and putative metabolic oncogene. J Oncol. 2014;2014:524101.

13. Guo W-b, Yang C, Bian J, Xia H, Yang J-k, Zhou Q-z, Chen M-k, Xue K-y, Zhang W-s, Wang P, Li X, Lui C-d. With a new clip technique surgically inducing varicocele in Sprague-Dawley rats. BMC Urol. 2018;18:58.

14. Velez de la Calle JF, Rachou E, le Martelot MT, Ducot B, Multigner L, Thonneau PF. Male infertility risk factors in a French military population. Hum Reprod. 2001;16:481-6.

15. Girgis SM, Abd el-Rahman Y, Awad H, Eisa I, Younan N, Mittawy B, el-Saleh QA. Lactate and pyruvate levels in the testicular vein of subfertile males with varicocele as a test for the theory of underlying hypoxia. Andrologia. 1981;13:16-9.

16. Ibrahim AA, Hamada TA, Moussa MM. Effect of varicocele on sperm respiration and metabolism. Andrologia. 1981:13:253-9.

17. Sofikitis N, Miyagawa I. Bilateral effect of unilateral varicocele on testicular metabolism in the rabbit. Int J Fertil Menopausal Stud. 1994;39:239-47.

18. Martins AD, Alves MG, Simões VL, Dias TR, Rato L, Moreira PI, Socorro S, Cavaco JE, PF O, research t. Control of Sertoli cell metabolism by sex steroid hormones is mediated through modulation in glycolysis-related transporters and enzymes. Cell Tissue Res. 2013;354:861-8.

19. Yao B, Zhou WL, Han DY, Ouyang B, Chen X, Chen SF, Deng CH, Sun XZ. The effect of the degree of left renal vein constriction on the development of adolescent varicocele in Sprague-Dawley rats. Asian J Androl. 2016;18:471-4.

20. Cho HM, Bae MA, Ahn JD, Kim YHJG. Nucleotide sequence and differential expression of the human 3-phosphoglycerate dehydrogenase gene. Gene. 2000;245:193-201. 
21. Xian Y, Zhang S, Wang X, Qin J, Wang W, Wu H. Phosphoglycerate dehydrogenase is a novel predictor for poor prognosis in gastric cancer Onco Targets Ther. 2016;9:5553-60.

22. Possemato R, Marks KM, Shaul YD, Pacold ME, Kim D, Birsoy K, Sethumadhavan S, Woo H-K, Jang HG, Jha AK. Functional genomics reveal that the serine synthesis pathway is essential in breast cancer. Nature. 2011; 476:346.

23. Choi JS, Kim IW, Hwang SY, Shin BJ, Kim SK. Effect of 2, 3, 7, 8tetrachlorodibenzo-p-dioxin on testicular spermatogenesis-related panels and serum sex hormone levels in rats. BJU Int. 2008;101:250-5.

24. Edassery SL, Shatavi SV, Kunkel JP, Hauer C, Brucker C, Penumatsa K, Yu Y, Dias JA, Luborsky JL. Autoantigens in ovarian autoimmunity associated with unexplained infertility and premature ovarian failure. Fertil Steril. 2010;94: 2636-41.

25. Zhu SM, Rao T, Yang X, Ning JZ, Yu WM, Ruan Y, Yuan R, Li CL, Jiang K, Hu W. Autophagy may play an important role in varicocele. Mol Med Rep. 2017;16:5471-9.

26. Wu H, Lin J, Liu P, Huang Z, Zhao P, Jin H, Ma J, Wen L, Gu N. Reactive oxygen species acts as executor in radiation enhancement and autophagy inducing by AgNPs. Biomaterials. 2016;101:1-9.

27. Shoji-Kawata S, Sumpter R, Leveno M, Campbell GR, Zou Z, Kinch L, Wilkins AD, Sun Q, Pallauf K, MacDuff D. Identification of a candidate therapeutic autophagy-inducing peptide. Nature. 2013;494:201-6.

28. Wang H, Sun Y, Wang L, Xu C, Yang Q, Liu B, Liu ZJ. Hypoxia-induced apoptosis in the bilateral testes of rats with left-sided varicocele: a new way to think about the varicocele. J Androl. 2010;31:299-305.

29. Razi M. Varicocele-induced infertility in animal models. Int J Fertil Steril. 2015;9:141.

\section{Publisher's Note}

Springer Nature remains neutral with regard to jurisdictional claims in published maps and institutional affiliations.

Ready to submit your research? Choose BMC and benefit from:

- fast, convenient online submission

- thorough peer review by experienced researchers in your field

- rapid publication on acceptance

- support for research data, including large and complex data types

- gold Open Access which fosters wider collaboration and increased citations

- maximum visibility for your research: over $100 \mathrm{M}$ website views per year

At $\mathrm{BMC}$, research is always in progress.

Learn more biomedcentral.com/submissions 